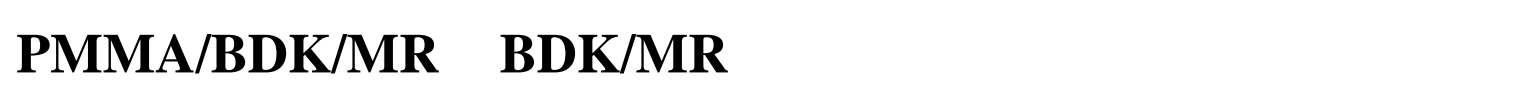 بلستخدلمهليفية النعاع الناdص
}

\author{
طa مصاف خضر المول \\ قنم الفيزياء \\ كلية العلوم \\ جلمعة الموصل
}

(تاريخ الاستلام 24/ 6 / 2008 ؛ تاريخ القبول 13/ 2009 )

\section{الملخ ص}

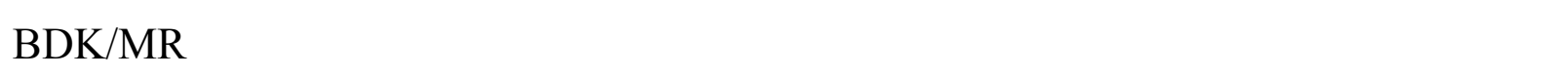

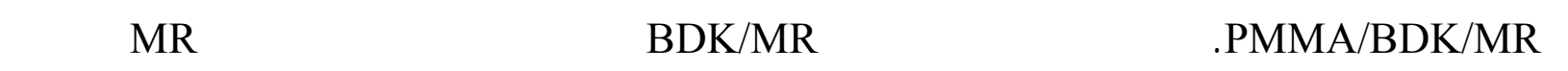
مخل BDK مثل (Azo - dye)

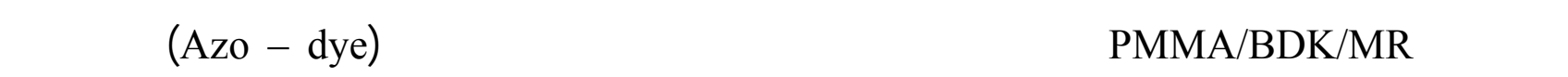

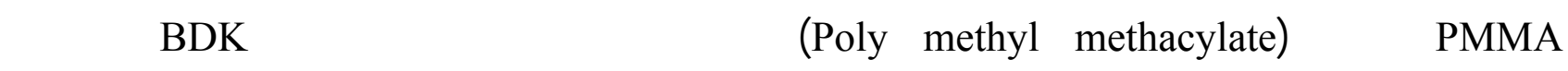

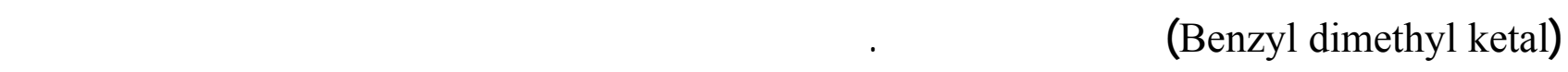

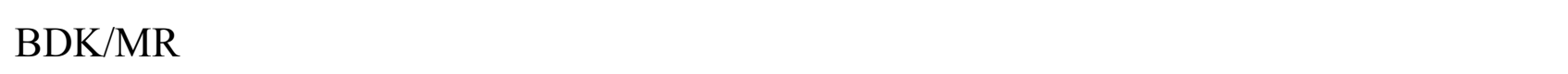
لكبر بكثير من الظلم الخزن البصري PMMA/BDK/MR ومن هنا نستنتج بلن الأغثية الرقيقة المحضرة من الظلم PMMA/BDK/MR أفضل من الأغشية الرقيقة المحضرة من الظلم BDK/MR وإضلفة هـ ـذه المعلوملت إلى المكتبة البرمجية لجهاز المطيفية.

الكاملت الدالة : تأثير الرطوبة، الأغشية الرقيقة، المكتبة البرمجية، جهاز المطيفية . 


$$
\text { طa مaطف خضر الموله }
$$

\title{
The Effect of Humidity on the Optical Storage BDK/MR and PMMA/BDK/MR Systems Using the Spectroscopic Eillpsometry
}

\author{
Taha. M. AL - Maula \\ Department of Physics \\ College of Science \\ Mosul University \\ tahamkader@yahoo.com.
}

\begin{abstract}
The effect of humidity on the two optical storage systems PMMA/BDK/MR, BDK / MR has been studied by preparing the system of BDK/MR from the Azo - dye of the methyl red (MR) and it is mixed with benzyl dimethyl ketal (BDK) in certain rates of size. But the other system PMMA/BDK/MR has been prepared from the Azo - dye of methyl red (MR) doped with poly methyl methacylate (PMMA) benzyl dimethyl ketal (BDK) and was used as a photoinitiartor at certain rates of size. The effect of humidity has been studied by using device of spectroscopical Eillipsometry which has different angles on the two systems of optical storage, and its become clear that the effect of humidity on the system BDK/MR is greater than that of PMMA/BDK/MR. From this we conclude that the thin films which were prepared from PMMA / BDK / MR system are better than those of the BDK/MR system and adding these information to the programming library for spectroscopical device .
\end{abstract}

Keywords: Effect of Humidity, Thin films, Programming library, Spectroscopical device

\section{المقدم - مة}

تعتبر درلسة تأثير الرطوبة للأغثية الرقيقة المكونة من صبغة الازو كمثل من المثل الأحم ـر MR

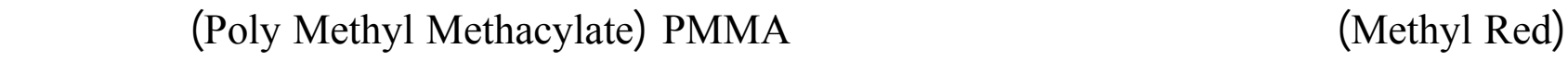
بالبادئ الضوئي Benzil Dimethyl Ketal) BDK) ذات أهمية كبيرة كونها تمل لحد الظوروف الخارجي ـة

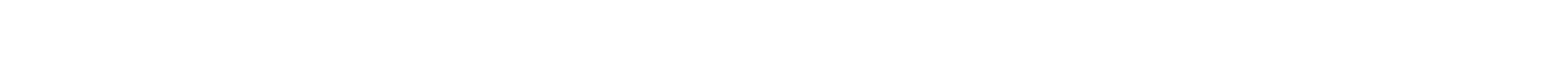

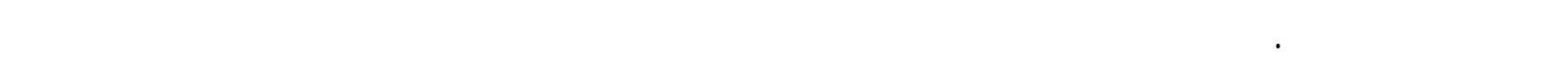

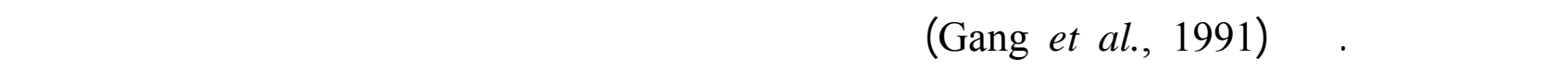

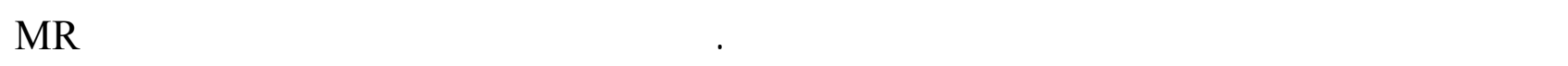

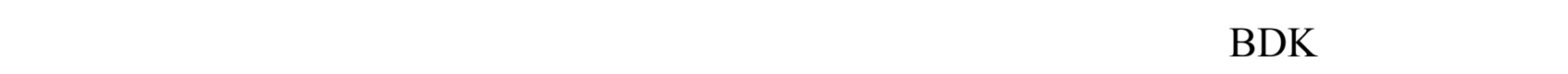
بالتشلكل الضوئي

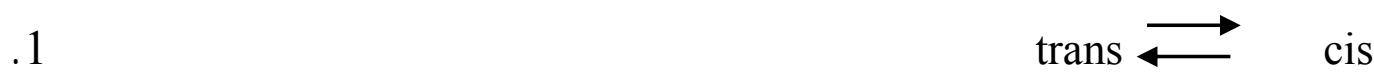




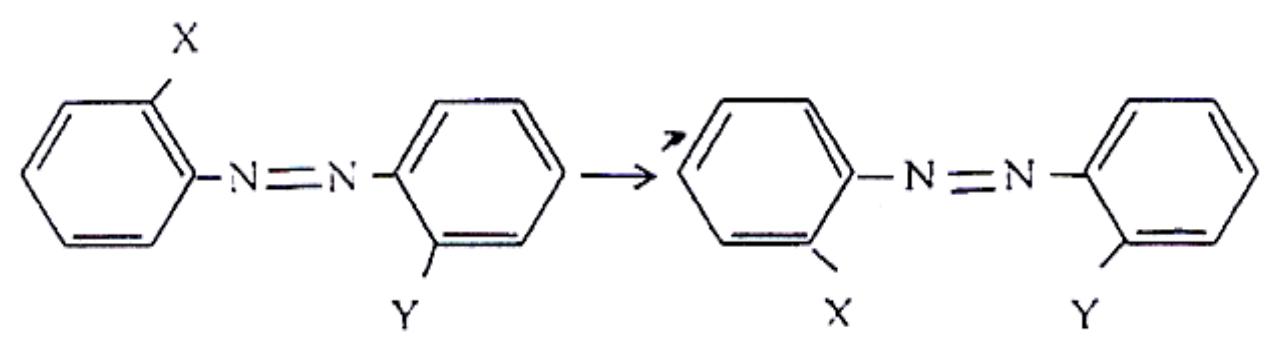

trans

Cis

للشكل 1 : التشلكل الضوئي لصبغة المثل الأحمر

أي لهم نفس التركيب وفف عدد الذرات لكنها تختلف في البنية الفراغية والتي تؤدي الم تغييرق ـيم

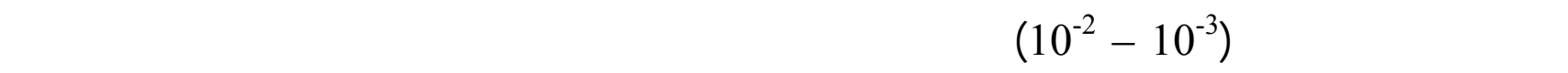

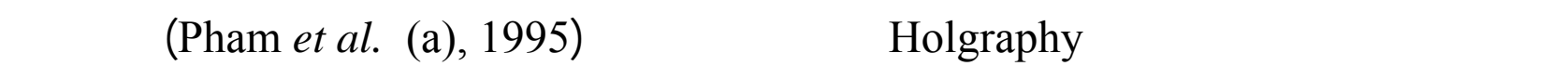
(AL - Attar et al., 2003) البنفجية كما في الثكل 2 .

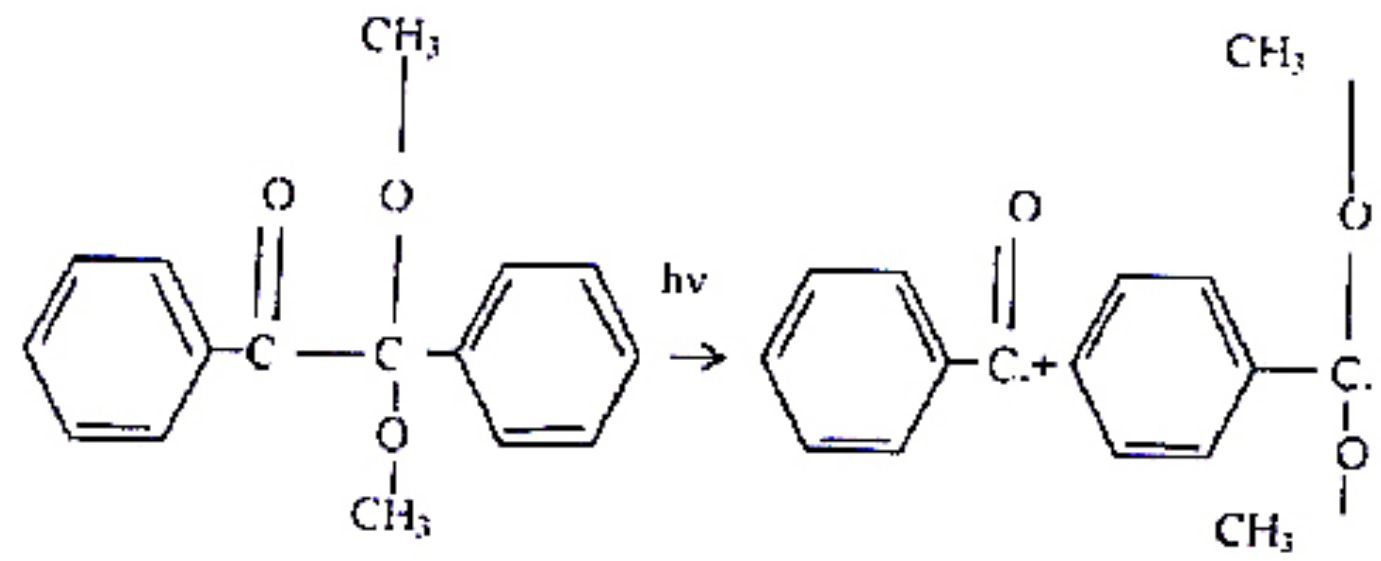

الشكل 2 : يبين قفكك BDK بوجود أشعة الفوق البنفجية

كما وجد (Day et al., 2001) أن البوليمر DaMA PM معلمل النكسار قليل ودرجة انصهار عالي ـة كما أن لهشففية عالية للضوء المرئي وخواص ميكانيكية مثالية .

\section{مواد طرق الهل}

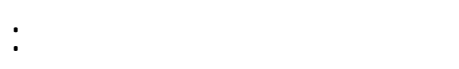
أولا : درلسة الرطوبة على ظلم الخزن البصري BDK / MR، قم تحضير محلول صبغة المثي لـ الأحم -ر MR وذلك بإضافة 0.5 a مسحوق المثل الأحمر إلى . ml 50 من مذيب الايثانول ورج ه جي دا وتشش يحه بولسطة ورق التشبح لأجل الحصول على محلول قي خالي من للثوائب . كما قم تحضير محلول الب ادئ 
الضوئي BDK وذلك بإضافة 0.5 a من مسحوق البادئ الضوئي إلى .

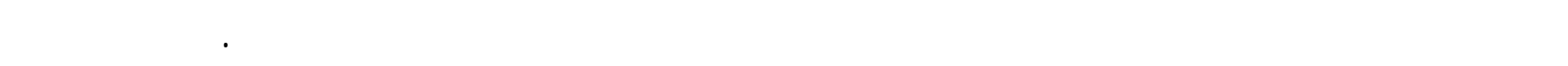
أغثية رقيقة من خليط مكون من BDK / MR بنسب حجمية معينة (3BDK: 1MR) وم تقظيف ال ششرائح

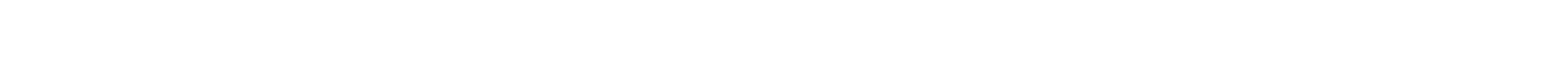

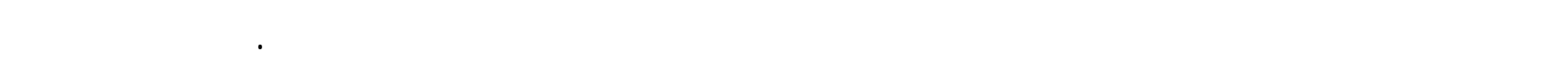
جيدا توضع كميلت معينة من مادة العينة المحضرة بولنطة الماصة الزجلجية ولجراء عملية الب ـرم ( Spin

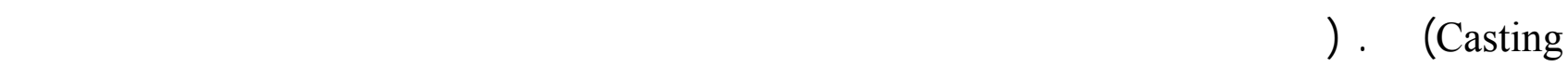

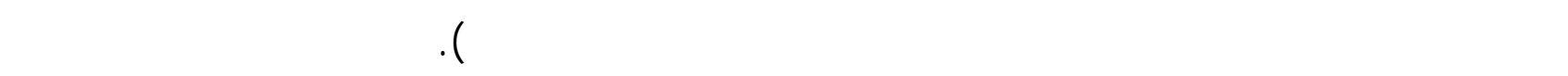

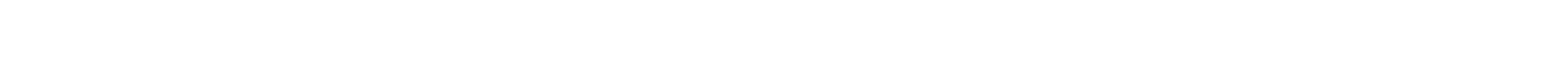

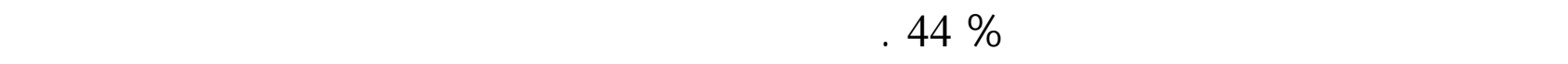

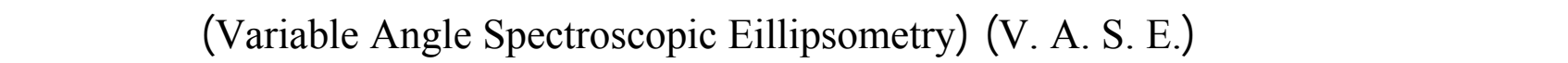
الأطول الموجية nm (1000 - 250) ولعددمن زوايا للسقوط (65, 70, 75) ومجهزة ببرمجي لت لإجج -راء

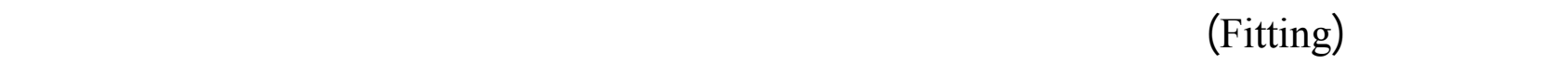

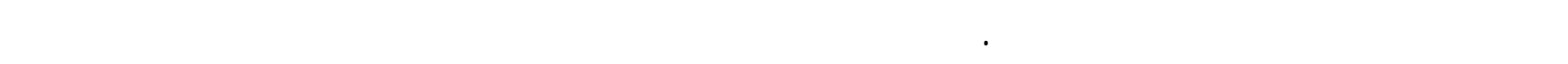
ويمر بعدها خ لل مـ ستنطب (Tompkins, 1999) ، (Azzam and Bashara, 1977) ، (un polarized)

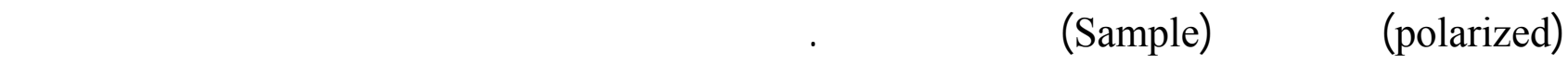

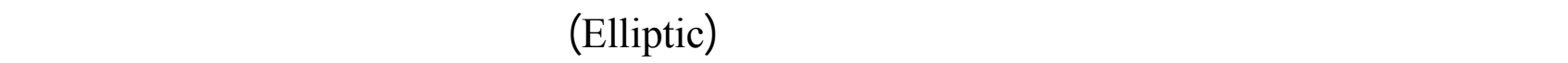
(Analyzer)

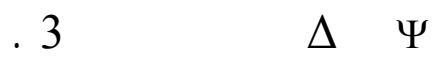

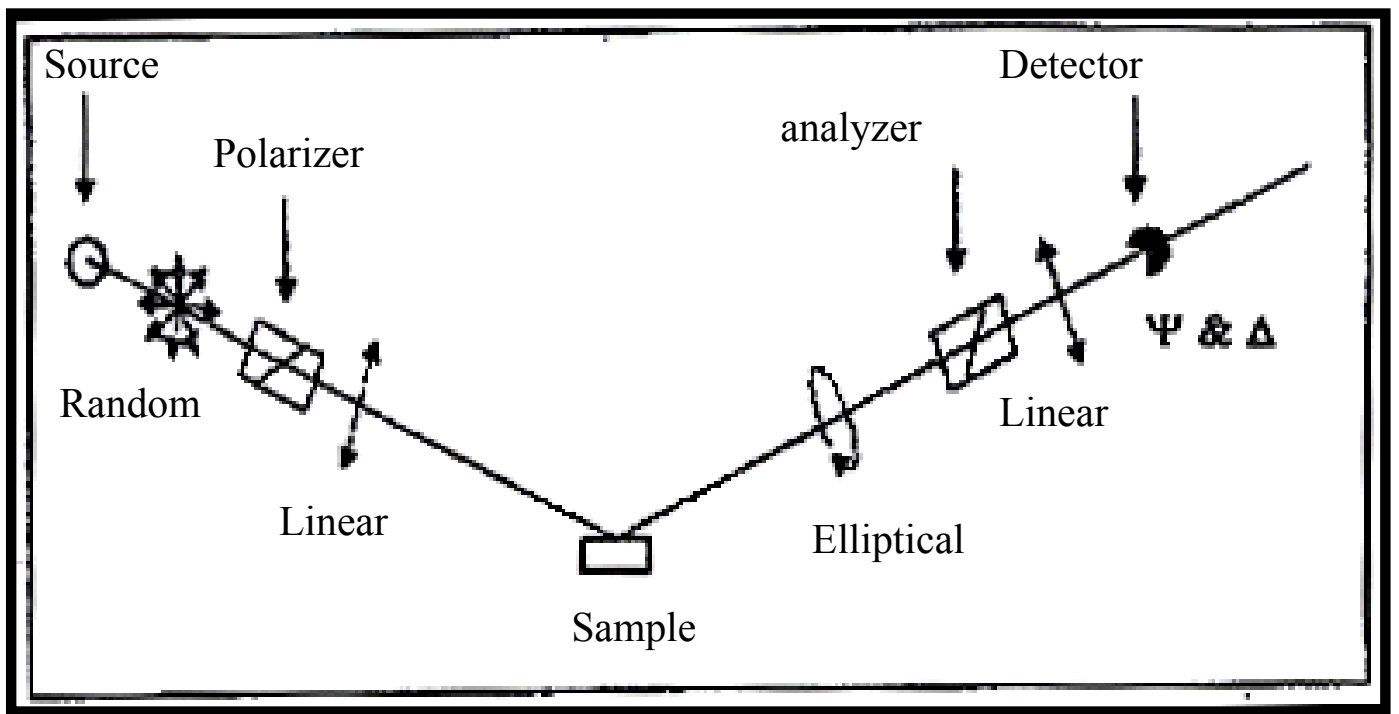

اللثكل 3 : خطوات القيلس بلستخدلم مطيافية التطع الناقص 


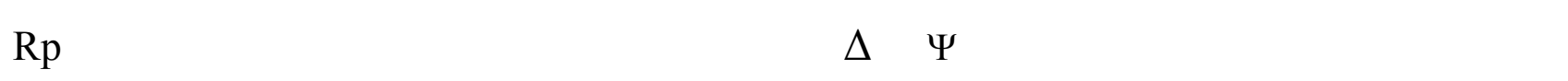
ss ،

$$
\begin{aligned}
\widetilde{\rho} & =\frac{\widetilde{R}_{p}}{\widetilde{R}_{s}} \\
\widetilde{\rho} & =\tan \psi \exp (i \Delta)
\end{aligned}
$$

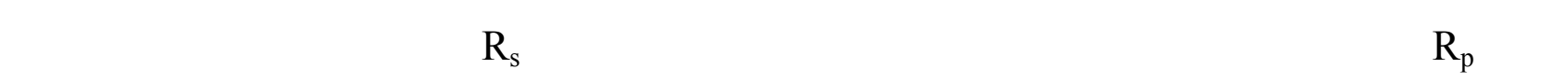
في حالة الإنظطلب العمودي، مَ النبة بين معلملي فريل العقدين للانعكلس في حالني الاسنطلب الموازي

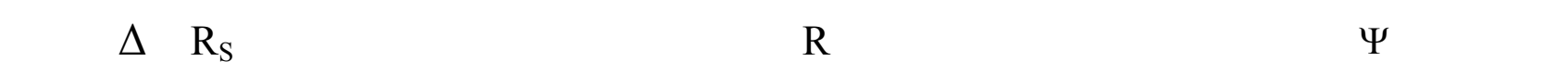

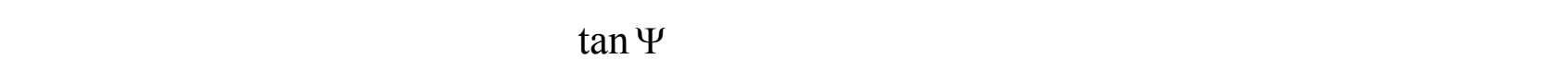

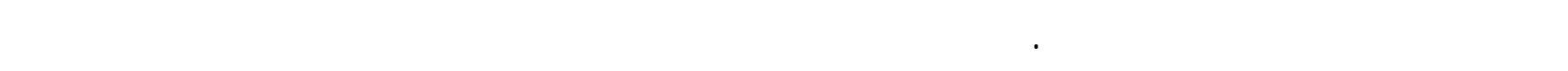

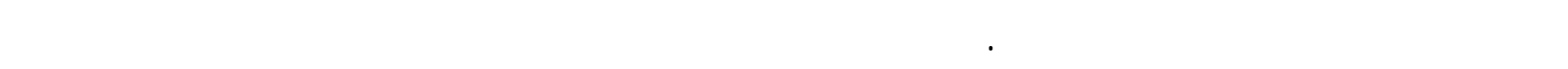

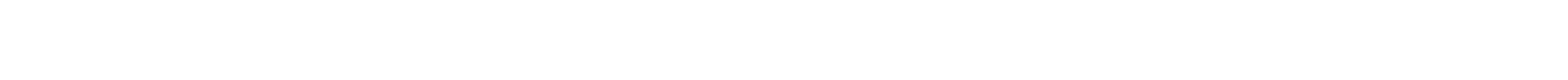

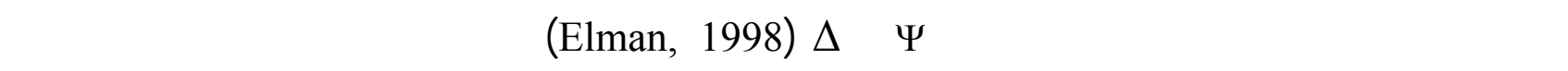

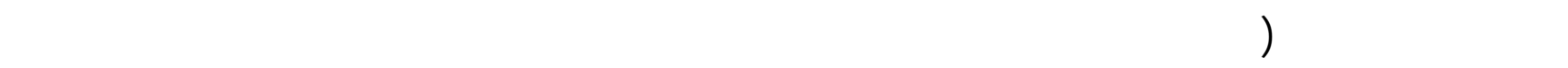

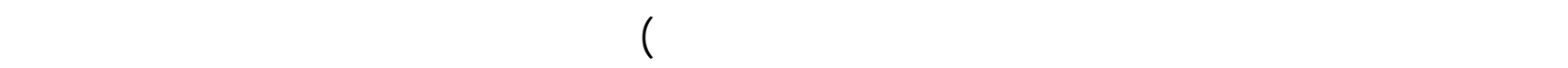

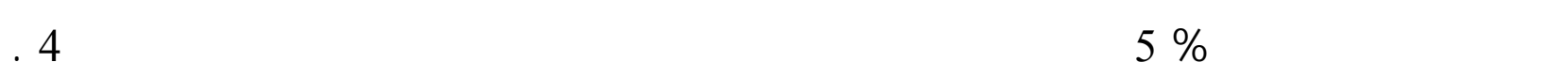

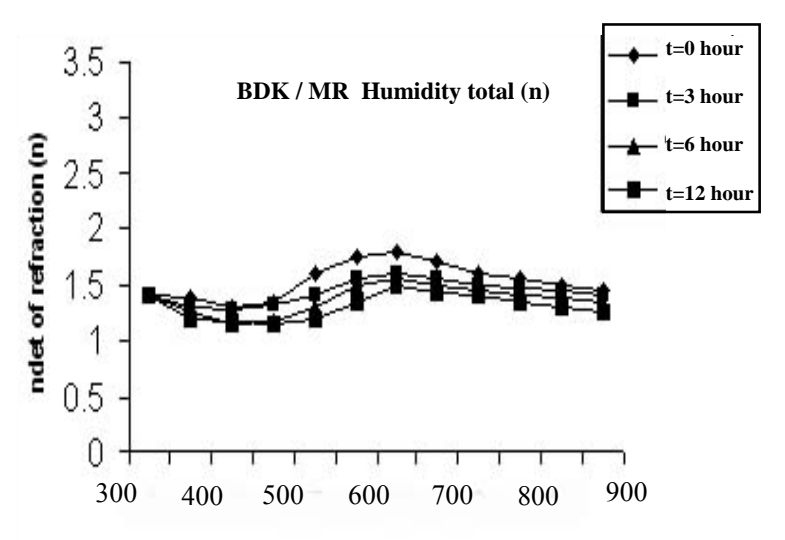

Wave length (nm)

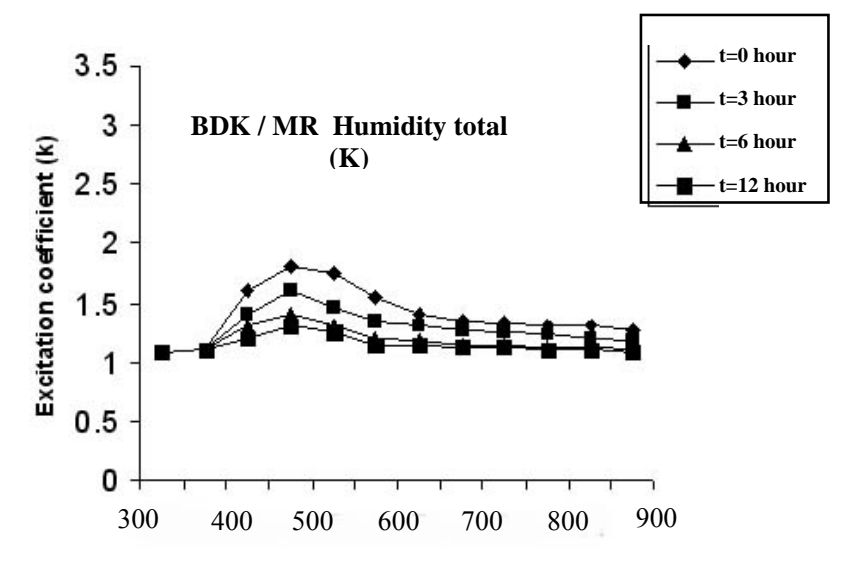

Wave length (nm)

اللثكل 4 : التغيير في معلمل الانكسار n ومعلمل الاظفاء k عند تغير الرطوبة للظلم BDK/MR

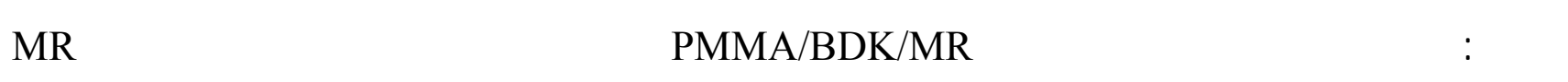

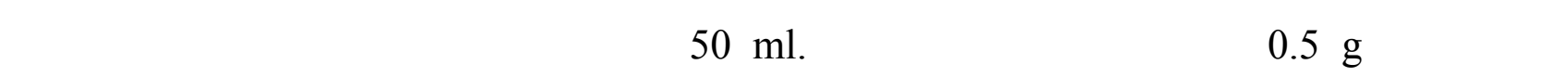


بولنطة ورق الترشبح لأبل الحصول على محلول قي خالي من الثشولئب ،وقم تح ضضير محل _ول الب ادئ

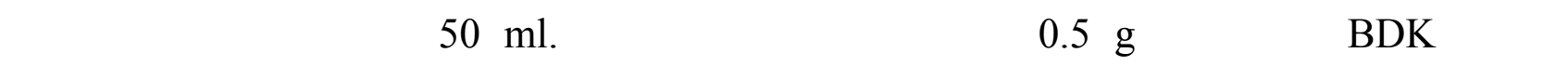

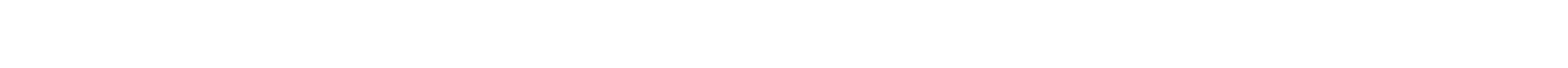

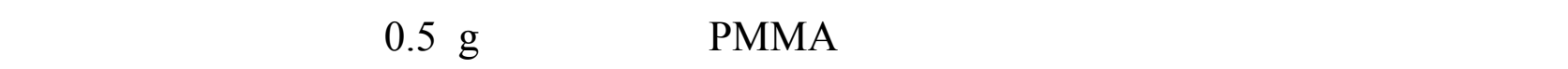

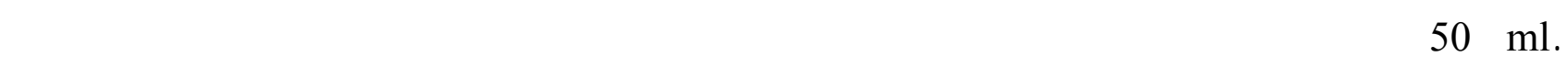

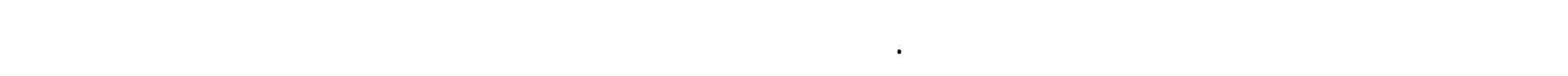

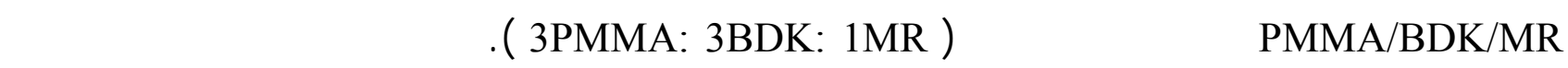

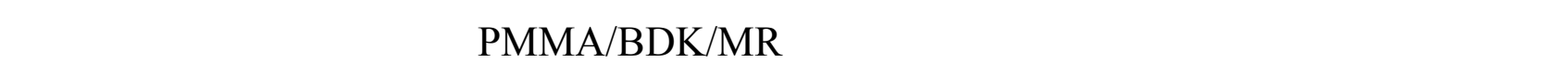

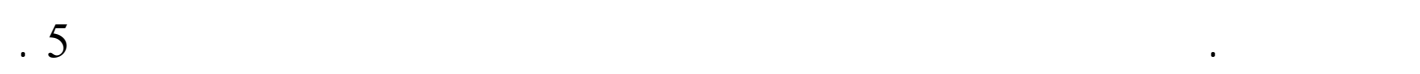
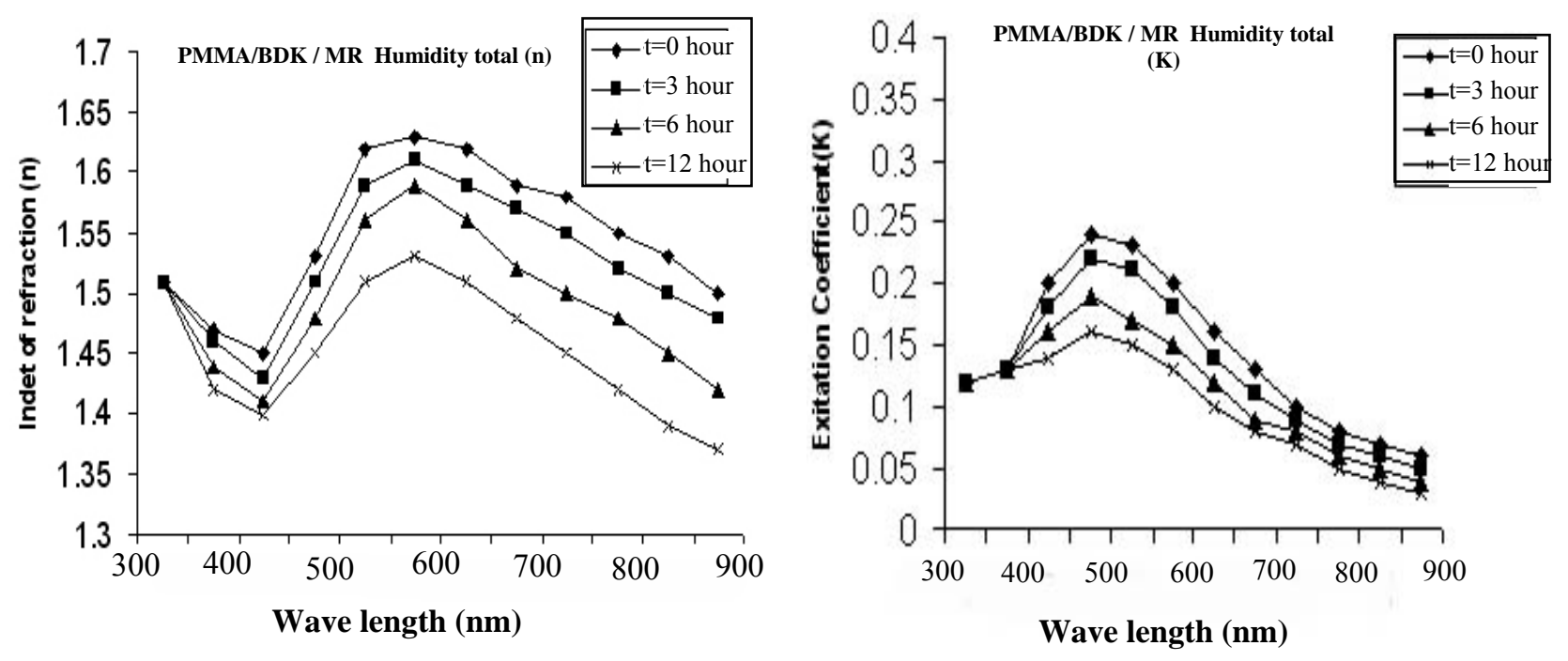

الثكل 5 : التغيير في معلمل الانكسار n ومعلمل الاظفاء k عند تغيير الرطوبة للظلم MMA/BDK/MR

\section{النتائج والمنالثشة}

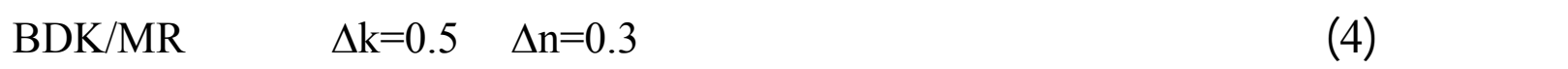

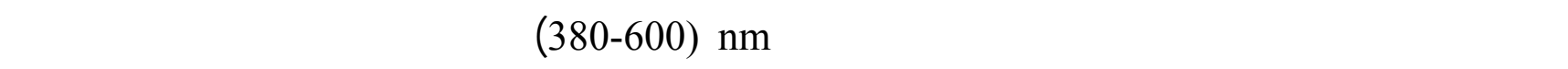

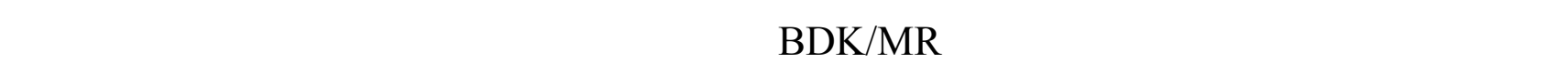

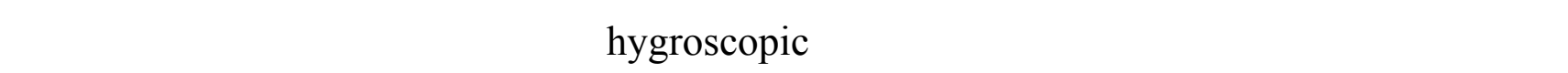

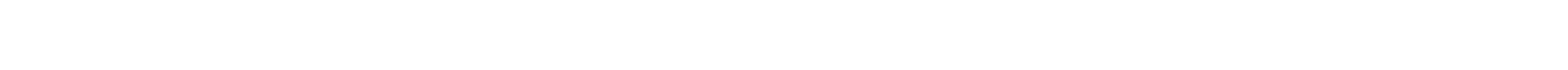

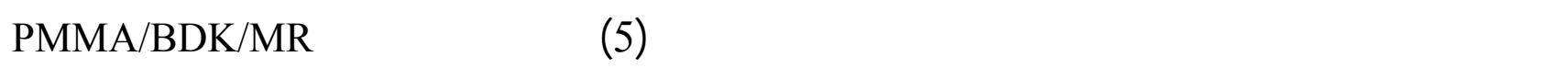


بالرطوبة فهو القل من ذلك الذي يحتوي على BDK/MR بالرغم من الانخفاض في المعملات البصرية ضمن المدى من الطوال الموجية المحدد من nm (600 -380 ) في المكل (5) عند تتع _رض الاغ ششية الرقيقة للرطوبة العالية ولفترة زمنية تصل اله 12ساعة الا ان الاغثية الرقيقة يظهر هبوط قلل في كلامن معلمل الانكسار مادة غير فعالة لذا تساهم جزيئل PMMA في اضغعلف القاعل بين الجذور الحرة الناتج ــ ع ـن قفكك البادئ الضوئي BDK ففع الضوء للستط على العينة التي عادة ما تهلجم الاصرة OH الموجوة ف ي

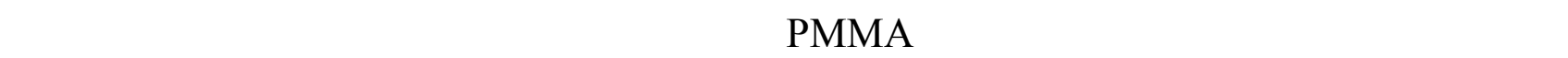
للظلمPPMA/BDK/MR. ويتبن من اللشكلين (4 ، 5) ان هنك تغير في قيم معلمل الانك سار للظ لمين

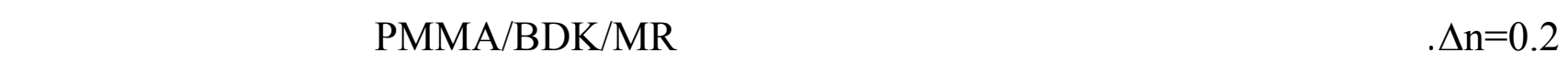
المحضرة من الظلم BDK/MR. وذلك لانسعة الخزن في الاغثية الرقيقة تكون كبيرة كلما كلن التغيير في قيم معلمل الانكسار صغيرة.

\section{لاستنتلحت}

من هذه الدرلسة مم التوصل إلى بعض النتائج المهمة :

1. الظلم BDK/MR ظظهر تأثيراكبيرا بالنبة للرطوبة وذلك نتيجة لامتصاص الماءمن قل الظله .

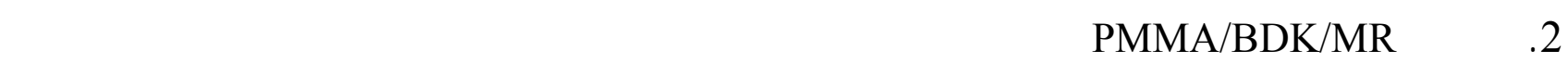

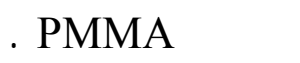

3. يفضل لستخدلم الظلم PMMA/BDK/MR بدلامن الظلم BDK/MR كوس ط خ _زن المعلوم ـات أو

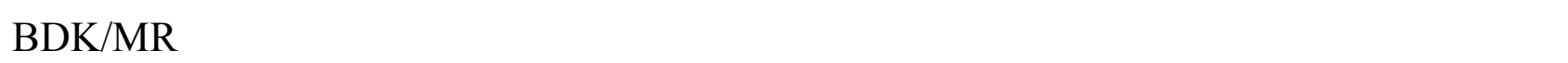
التي يكون تأثير الرطوبة عليها كبيرة جدا .

\section{المصاير الحرية}

MMA/BDK/Azo الموله،طه مصطف خضر،( 2005 ن). الخصائص البصرية لأظمة الخزن البصرية البرية

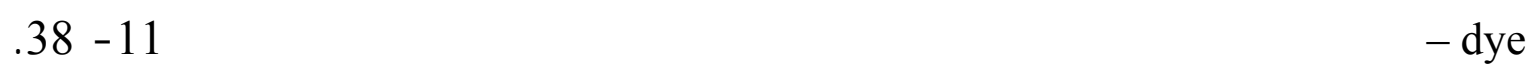
المصار الأجنبية

AL-Attar, H. ;Tagatqa, O. (2003). Pure and applied optics, J. of Optics. 5(6), 478 - 492. Azzam, R. ; Bashara, N. (1977). "Ellipsometery and Polarized Light". 1st edn, North Holland physics publishing, Amesterdam, $8 \mathrm{p}$.

Douglas, A. ; Donald, M. (1980). "Principles of Instrumental Analysis". 2nd edn Hatt , Saurders, Japan , $20 \mathrm{p}$. 
Elman, J. F.; Greener, J.; Greener, C. M. ; Hertinger, B. johs (1998). Characterization of bixially - stretched plastic films by generalized ellipsometry. J. of molec. struc. 313 (13), 814 - 818.

Gang, S. ; Xuchum, P. (1999). Study of the optical properties of thin solid films. Opt. Com, 564(20), 3049.

Pham, M. (1995). Modelling growth instability in astressed solid. Growth and Characterization of Materials, 9(10), $302-325$.

Tompkins, H.G.; Megaham, W. A. ( 1999). "Spectroscopic Ellipsometry and Reflectivity, A user's guide", Johm Wiley and Sons, New York. pp.70-71. 